\title{
Detection of Wavelet Transform-Processed Ventricular Late Potentials and Approximate Entropy
}

\author{
S Bunluechokchai, MJ English \\ University of Sussex, Brighton, UK
}

\begin{abstract}
Ventricular Late Potentials (VLPS) are low-level highfrequency signals that are usually found within the terminal part of the QRS complex from patients after Myocardial Infarction. Patients with VLPs are at risk of developing Ventricular Tachycardia, which is the major cause of death of patients suffering from heart disease.

In this study, the Continuous Wavelet Transform was used to detect VLPS and then approximate entropy (ApEn) was applied to classify subjects with and without VLPS. Approximate entropy has been recently proposed to quantify the irregularity in physiological signals. High ApEn values indicate greater irregularity. Patients with VLPs tend to have higher ApEn values than those without. Preliminary investigations in this study show promising results for distinction between the two patient groups.
\end{abstract}

\section{Introduction}

Ventricular Late Potentials are low-amplitude, highfrequency signals that generally occur within the terminal part of the QRS complex and the beginning of the ST segment. It is believed that VLPs are generated from a region of the damaged myocardium where the ventricular depolarisation is delayed. VLPs appear as fractionated signals with irregularity in shape on the body surface. From the studies in clinical cardiology, it has been shown that the occurrence of VLPs is prevalent in postMyocardial Infarction (MI) patients at risk of developing Ventricular Tachycardia, which is one of the leading causes of sudden cardiac death. Hence the detection of VLPs has become a topic of great interest in cardiology for over three decades. Early diagnosis of heart disease by detecting VLPs might save a large number of patients' lives.

The standard method for detecting VLPs was proposed by Simson [1,2]. This method computes the parameter measurements obtained from the filtered signal-averaged ECG in the time domain. Many attempts have been made to study VLPs using time-frequency domain techniques, for example the Wigner Distribution (WD) and the Short Time Fourier Transform (STFT). The limitation of the WD is that it produces unwanted interference terms that do not reflect the original signal whereas the STFT has fixed time-frequency resolution that is not optimal for the analysis of non-stationary signals. VLPs are assumed to be non-stationary signals. The wavelet transform is proposed as an alternative technique and it can overcome the limitations encountered in the WD and STFT.

In this study, the Continuous Wavelet Transform (CWT) was used to analyse the High Resolution ECG (HRECG) in the time-scale domain. Then, based on the CWT, a new concept of approximate entropy was investigated to classify patients with and without VLPs.

\section{Data acquisition}

High Resolution ECG recordings were acquired from post-MI patients using orthogonal XYZ leads at a sampling rate of $1000 \mathrm{~Hz}$ and a resolution of 12 bits. The standard time domain method [1,2] was used to identify patients who exhibited the presence of VLPs. According to the standard method, the patient data set was divided into two groups, patients with and without VLPs. The results of the two patient groups were taken as a reference to evaluate the proposed method in this study.

\section{The continuous wavelet transform}

More recently, among time-frequency methods, the CWT seems to be a tool of choice for HRECG analysis $[3,4]$. The main advantage of the CWT is that it provides variable time frequency resolution. It is defined as:

$$
C W T(\tau, a)=\frac{1}{\sqrt{a}} \int_{-\infty}^{\infty} s(t) \psi\left(\frac{t-\tau}{a}\right) d t
$$

where $s(t)$ is the signal, $\psi(t)$ is the mother wavelet, scaled by $a$ and shifted by $\tau$. The scaling parameter $a$ can be viewed as the inverse of frequency. Dilating or contracting the wavelet changes frequencies of interest. The wavelet is contracted at smaller scales, corresponding to high frequencies, which can detect high frequency components of the signal whereas the wavelet is dilated at larger scales, relating to low frequencies, which can extract the low frequency components of the signal. This provides varying time-frequency resolution. Shifting the wavelet is to localise the signal in time. The CWT thus offers a good compromise between time 
localisation and frequency resolution. Further details about the CWT can be found in the literature [5,6]. It is obvious that the variable time frequency resolution characteristic of the CWT is well suited to the analysis of non-stationary signals containing short, high-frequency components. As VLPs are considered to be short-duration high-frequency signals, the CWT seems to be a promising technique for this task. The CWT with smaller scales can be successfully used to detect the presence of VLPs [7]. Because the CWT is computed in terms of scale instead of frequency, it produces a time-scale representation of the signal.

\section{HRECG analysis with the CWT}

To detect the presence of VLPs, the CWT with small scales was used. Each individual signal XYZ lead was first averaged to reduce the random noise level and each of the averaged XYZ leads was then applied to the CWT. The CWT of scales 3-10 using a Daubechies 2 mother wavelet was investigated. The CWT from each lead was then combined to form a vector magnitude CWT as defined in Equation 2. This allows all of the late potential information to be analysed.

$$
|C W T V|=\sqrt{\left(C W T X^{2}+C W T Y^{2}+C W T Z^{2}\right)}
$$

where $C W T V$ is the vector magnitude. $C W T X, C W T Y$, and $C W T Z$ are the CWT of the XYZ leads, respectively.

The resulting vector magnitude $C W T V$ plots for scales 3-10 from a patient without VLPs and another patient with at the time interval from the $\mathrm{R}$ wave to $150 \mathrm{~ms}$ after are illustrated in Figures 1 and 2, respectively. It appears that the vector magnitude $C W T V$ is becoming increasing regular from scales 3 to 10 in both patients. By visual inspection in Figures 1 and 2, an observable difference between the two patients can be obtained. The patient with VLPs exhibits greater fluctuation or irregularity than the one without VLPs over scales 3 to 10.

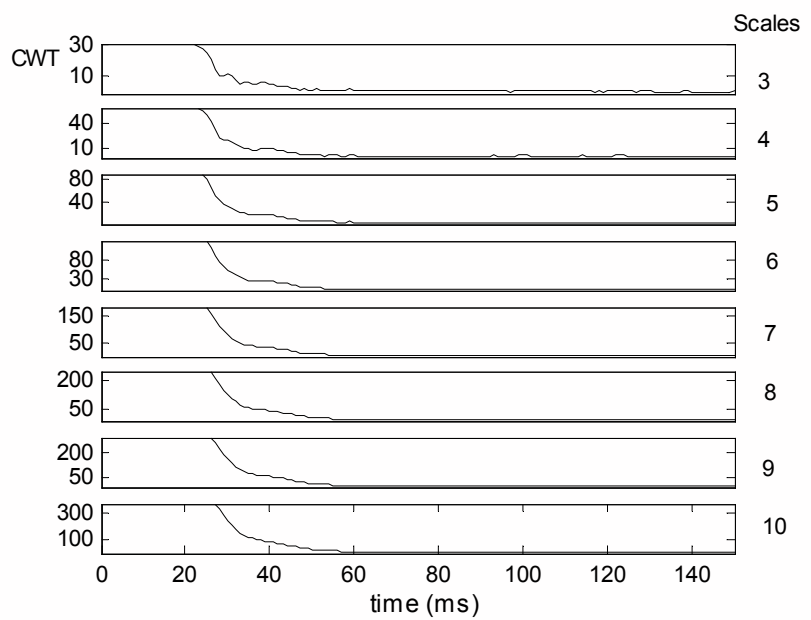

Figure 1. The $C W T V$ plot for a patient without VLPs at scales 3-10.

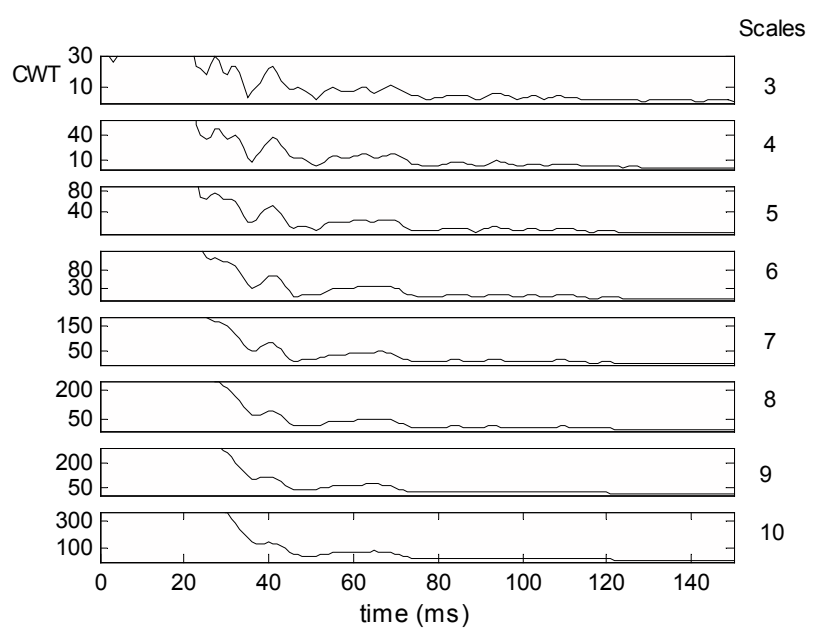

Figure 2. The $C W T V$ plot for a patient with VLPs at scales 3-10

\section{Approximate entropy}

There is increasing interest in measuring the degree of irregularity of physiological data using Approximate Entropy (ApEn). ApEn has shown a very significant difference in irregularity of physiological measurements between normal and abnormal patients. It has been applied widely to heart rate variability, changes in hormone levels, and EEG analysis.

Pincus first proposed approximate entropy as a regularity statistic for system complexity [8]. It is a new mathematical approach, which can be used to quantify the irregularity of signals. Signals containing regular patterns (e.g. sinusoidal signals) have small ApEn values whereas those with irregular behaviour (e.g. random noise) show high ApEn values.

In this study, ApEn is slightly modified and defined as follows $[8,9]$ :

1. Given data $u(n)$ (e.g. ECG data) with $\mathrm{N}$ data points,

$u(n)=u(1), u(2), u(3), \ldots, u(N) ; n=1,2,3, \ldots, N$

2 . Form vector sequences of length $m$ from the given data $u(n)$ : Vector sequences $x(1), \ldots, x(N-m+1)$ are formed by defining:

$x(i)=\lfloor u(i), u(i+1), \ldots, u(i+m-1)] ; i=1, \ldots, N-m+1$

3. Define the distance $d[x(i), x(j)]$ between two vectors $x(i)$ and $x(j)$ as the maximum absolute difference between their corresponding scalar components.

$d[x(i), x(j)]=\max [u(i+k)-u(j+k)]] ; k=0, \ldots, m-1(5)$

4. For a given vector $x(i)$, count the number of $j$

such that $d[x(i), x(j)] \leq r$

The parameter $r$ is the factor of similarity that acts like a noise filter.

Let $M(i)$ be the number of $j$ such that $d[x(i), x(j)] \leq r$. 
Then $C_{r}^{m}(i)=\frac{M(i)}{N-m+1} ; i=1, \ldots, N-m+1$

Two vectors, $x(i)$ and $x(j)$, are similar if $d[x(i), x(j)] \leq r$. Thus, $M(i)$ is the number of vectors in all $N-m+1$ vectors, which are similar to a given reference vector $x(i)$. The quantity $C_{r}^{m}(i)$ is the probability that any vector $x(j)$ within the distance $r$ is similar to a given vector $x(i)$ of length $m$, therefore $C_{r}^{m}(i)$ of each vector can be computed. Then, $P_{m}(r)$ is defined as the mean of all the $C_{r}^{m}(i)$ values.

5. Increase the length to $m+1$, repeat steps $2-4$ and find $C_{r}^{m+1}(i), P_{m+1}(r)$.

6. Calculate $\operatorname{ApEn}(m, r)$ :

$$
\operatorname{ApEn}(m, r)=\ln \left[P_{m}(r) / P_{m+1}(r)\right]
$$

ApEn provides a measure of irregularity. A signal with high regularity has a very small ApEn value. On the other hand, a signal with very low regularity produces a high ApEn value. In computing ApEn, two input values, $m$ and $r$, must be specified. As suggested by Pincus, the most commonly used $m$ value is 2 for physiological data. The $r$ value should be at least 3 times the mean noise amplitude in order to prevent computation error of the ApEn value [8].

\section{Quantifying the HRECG irregularity}

To quantify irregularity of the $C W T V$ plots in Figures 1 and 2, the approximate entropy method described in Section 5 was used. ApEn was applied to the vector magnitude $C W T V$ and then the ApEn value was computed for each patient. The vector magnitude obtained from the CWT was investigated at different time intervals for computation of the ApEn value. Time intervals started from the $\mathrm{R}$ wave, $10 \mathrm{~ms}$ after, $20 \mathrm{~ms}$ after, $30 \mathrm{~ms}$ after, and $40 \mathrm{~ms}$ after the $\mathrm{R}$ wave.

\section{Results of ApEn}

For each patient, the ApEn value of each scale from 310 was computed at different values of $m$ and $r$ for different time intervals. The $m$ values of 1,2 and 3 were tested and the $r$ values of 3-4 times the mean absolute noise, in steps of 0.1 , were examined. The mean absolute noise was calculated at the ST segment where no cardiac activity was present.

It was found from the results that greater irregularity of VLPs were obtained in the small scales of the CWT at the $m$ value of 2 and $r$ of 3.2. In this study, scales 3, 4, and 6 were used for the ApEn calculation because of the distinguishing difference between the two patient groups. The mean ApEn obtained from the scales 3, 4, 6 was computed at different time intervals. The computed mean ApEn at the time duration from 20 and $30 \mathrm{~ms}$ after the $\mathrm{R}$ wave revealed distinct separation between patients with and without VLPs. For example, the computed mean ApEn of a patient without VLPs was 0.004 and 0.031 for a patient with VLPs at the time duration of $30 \mathrm{~ms}$ after the $\mathrm{R}$ wave. Results of the computed mean ApEn values for the time durations of 20 and $30 \mathrm{~ms}$ after the $\mathrm{R}$ wave are shown in Figures $3 a$ and $3 b$, respectively. In Figures $3 \mathrm{a}$ and $3 \mathrm{~b}$, the difference between the two patient groups was confirmed at the $99.9 \%$ level of confidence $(p<0.0005)$ from the Wilcoxon Rank Sum Test. Patients are classified as borderline patients if their measurement parameters computed from the filtered HRECG are very close to the criteria values of the Simson's standard method.

In Figure 3a, a threshold value of 0.0203 was empirically chosen to differentiate the two patient groups. The sensitivity and specificity of the ApEn were computed for the complete and modified data sets. The results were sensitivity of $85 \%$ and specificity of $96 \%$ for the complete data set. Sensitivity of $100 \%$ and specificity of $100 \%$ were obtained in the modified data set where the borderline patients are removed.

In Figure 3b, the sensitivity and specificity are the same as those in Figure 3a for both the complete and modified data sets at the empirically chosen threshold value of 0.0168 .

In Figure 3, It is interesting to note that there are a few patients with and without VLPs who were possibly misclassified by the Simson method. When these misclassified patients were removed, the sensitivity and specificity were recomputed. Figures $3 \mathrm{a}$ and $\mathrm{b}$ show the same recomputed sensitivity of $94 \%$ and same recomputed specificity of $98 \%$ for the complete data set.

a
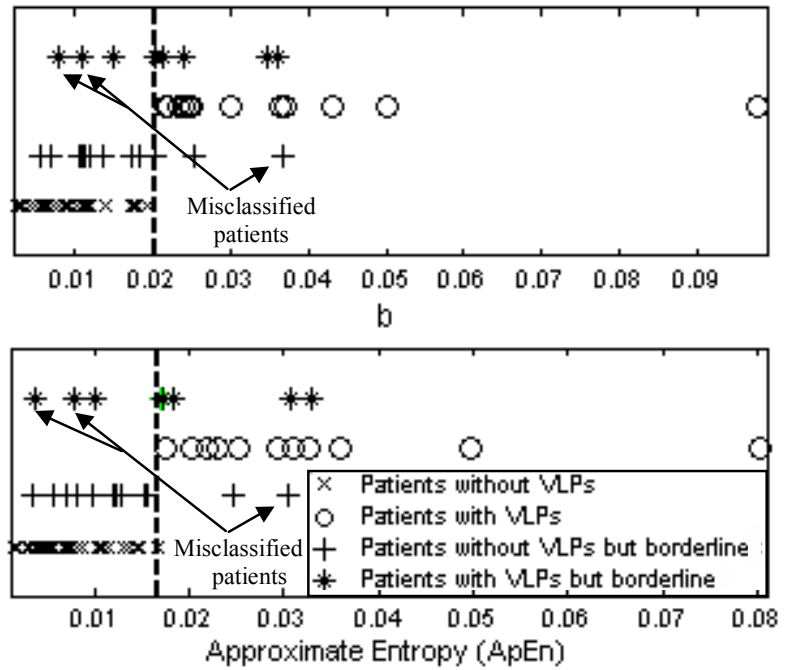

Figure 3. The computed ApEn from the time interval 20 $\mathrm{ms}$ after the $\mathrm{R}$ wave (a) and $30 \mathrm{~ms}$ after (b). 


\section{Discussion and conclusions}

A new concept of approximate entropy was applied to the CWT processed HRECG data. The approximate entropy has been widely used to quantify the subtle difference in regularity of signals. Greater irregularity of a signal produces a larger ApEn value. Patients with VLPs are more irregular whereas patients without VLPs are more regular. As expected, patients with VLPs have larger ApEn values than those without VLPs. The ApEn value may provide additional findings that patients with VLPs would produce highly irregular signals from their damaged myocardium.

In this study, different time intervals of HRECG data from the $\mathrm{R}$ wave were investigated. Results showed that the significant difference between two patient groups started detecting irregularity at a time of $20 \mathrm{~ms}$ after the $\mathrm{R}$ wave. One of the possible reasons is that the high QRS energy would mask the low level VLPs between the R wave and just before $20 \mathrm{~ms}$ after the $\mathrm{R}$ wave. Another reason is that VLPs with the CWT analysis would start occurring at $20 \mathrm{~ms}$ after the $\mathrm{R}$ wave.

In computing ApEn, two parameters, $m$ and $r$ must be fixed. In this work, $m$ of 2 was selected due to the best performance obtained and this value was widely used with successful results in the clinical application. For the $r$ value, it was recommended that the $r$ value should be chosen to be larger than the noise, on the other hand, it should not be too large else the detailed information of VLPs would be lost. Results of ApEn exhibited that the appropriate value of $r$ should be 3.2 times the mean absolute noise.

\section{Acknowledgement}

The first author of this study was supported by a scholarship from the Thai government.

\section{References}

[1] Simson MB. Use of signals in the terminal QRS complex to identify patients with ventricular tachycardia after myocardial infarction. Circulation 1981;64(2):235-242.

[2] Breithardt $G$ et al. Standards for analysis of ventricular late potentials using high resolution or signal-averaged electrocardiography - A statement by a Task Force Committee between the European Society of Cardiology, the American Heart Association and the American College of Cardiology. European Heart Journal 1991;12:473-480.

[3] Jones DL, Touvannas JS, Lander P, Albert DE. Advanced Time-frequency Methods for Signal Averaged ECG Analysis. Journal of Electrocardiology 1992;25:188-194.

[4] Khadra L, Dickhaus H, Lipp A. Representations of ECG late potentials in the time frequency plane. Journal of Medical Engineering and Technology 1993;17:228-231.

[5] Bentley PM, McDonnell JTE. Wavelet transforms: an introduction. IEE Electronics and Communication Engineering Journal 1994;175-186.

[6] Rioul O, Vetterli M. Wavelets and Signal Processing. IEEE Signal Processing Magazine 1991;14-38.

[7] Spaargaren A, English MJ. Detecting Ventricular Late Potentials using the Continuous Wavelet Transform. IEEE Computers in Cardiology 1999;26:5-8.

[8] Pincus SM, Goldberger AL. Physiological time-series analysis: What does regularity quantify? Am J Physiol Heart Circ Physiol 1994;266,No. 4:H1643-H1656.

[9] Ho KKL, Moody GB, Peng CK et al. Predicting survival in heart failure case and control subjects by use of fully automated methods for deriving nonlinear and conventional indices of heart rate dynamics. Circulation 1997;96(3):842848.

Address for correspondence.

Dr. M.J. English

Department of Engineering \& Design

University of Sussex

Brighton BN1 9QT

UK

m.j.english@sussex.ac.uk 